\title{
ChilDiBu - A Mobile Application for Bulgarian Children with Special Educational Needs
}

\author{
Radoslava Kraleva \\ Department of Informatics, South-West University “Neofit Rilski”, Blagoevgrad, Bulgaria \\ E-mail: rady_kraleva@swu.bg
}

\begin{abstract}
Designing applications adapted to the capabilities of each user is an important part of modern software engineering. Children with Autism spectrum disorder and/or intellectual disabilities are a special group of users where standard application design is not a good approach. Some of them have speech disorders, others have disorders of the musculoskeletal system and in their cognitive development. In the literature, these children are referred to as children with special educational needs.

Only few researchers are involved in the development of various computer technologies, including mobile applications appropriate for these children. Some specialized mobile applications for these users can be found in the different mobile stores. However, most of these apps are rather expensive or with inappropriate user interfaces. Another disadvantage in all the apps is the lack of support in the Bulgarian language. It makes them unusable for the youngest users with special needs in Bulgaria. A conceptual model based on the investigation of some existing applications, the well-established theoretical practices, and on the opinion of children without disorders is proposed in this paper. An approach to designing and developing the interactive mobile application ChilDiBu for children with special needs, suitable for the Windows Phone mobile operating system, is presented. ChilDiBu is a picture-based app that combines graphic images, texts and audio files which can be used to learn the Bulgarian alphabet, the numbers up to 20 , some basic colors and the daily activities that children perform. The application is focused on the development of the fine motor skills, observation and decision-making skills which is completed with a puzzle for arranging the two-digit numbers. At the end of the paper the app interface, the way it is used and its app architecture are shown.
\end{abstract}

Keywords - children with cognitive disorders; mobile application; mobile development; picture-based apps; child-computer interaction; software engineering

\section{INTRODUCTION}

Designing applications adapted to the capabilities of each user is an essential activity of modern software engineering. Children with spectrum disorder and/or intellectual disability are a special group of users where standard application design is not a good approach. Nowadays figures show that more than a billion people, including children, have some disabilities [1]. This negative phenomenon leads to the need of overcoming some constraints that accompany the lives of both patients and their relatives. New technologies and interactive tools are a good way to support young children's cognitive, social, emotional, physical, and linguistic development [2].

Before delving into the main issues of this article, the concepts of children with disabilities and special educational needs must be defined. According to UNICEF [3], disability can occur at three levels: an impairment in body function or structure, a limitation in activity, and a restriction in participation. "Children with disabilities include those with health conditions such as cerebral palsy, spina bifida, muscular dystrophy, traumatic spinal cord injury, Down syndrome, and children with hearing, visual, physical, communication and intellectual impairments" [3]. Often, they have disorders in the speech, the musculoskeletal system and in their cognitive development. For example, children with Autistic Spectrum Disorders (ASD) have limitations in the perception of the outside world, limited social relationships, difficulty in concentrating on individual stimulus, lack of organizational skills, and so on [4], [5], [6]. Typically, these children also have special educational needs, and standard training methods and systems are impracticable without prior adaptation.

For children suffering from mental disorders, such as Autism, the support and training start from early childhood. In most countries, specialized prescriptions and programs have been developed for this purpose. Early learning is often done with the help of associations with visual objects and visual supports in the form of playing cards or other realworld objects that children can touch and feel. However, parents and teachers still encounter difficulties in providing and using them [7]. 
The use of a mobile device does not require keyboarding, a button is not pressed to start, restart or shutdown a computer and a monitor, nor is a computer mouse needed. The interaction is possible only by touching the sensitive screen. All children, whether disabled or not, study the surrounding world by touching, feeling, crushing, etc. Therefore, smartphones and tablets (also named mobile devices) are some of the preferred and affordable technologies for children. They quickly learn to use them for fun, communication, training, and so on [8], [9]. Although it is a relatively new direction in computer technology, the large use of modern mobile technologies, such as tablets and smartphones, in the daily lives of people with disabilities enhances rapidly. The need for more detailed scientific research related to their evaluation, usefulness and disadvantages is still an object of research [10].

Few researchers are involved in developing various computer technologies, including software products appropriate for children with disorders. The different mobile stores such as Aacorn AAC [11], AraBoards [12], Autism Therapy with MITA [13], offer a certain number of such applications. At the moment of the present study, all similar apps examined did not have a Bulgarian language support which makes them inapplicable to children in Bulgaria. In addition, a large part of them are costly or have very limited functions. In other cases, one can note, that the apps' interface does not conform to children's preferences and abilities.

This article suggests an approach for designing and developing mobile applications targeting children with Autism spectrum disorders and/or intellectual disability, including those with special educational needs. This approach is based on a previous study regarding the design of an interface for a mobile application based on children's opinion presented in [14]. The pilot mobile application ChilDiBu has been developed with a Bulgarian language support for children with special educational needs and is presented in the following sections of the paper. Also, a 9year child (girl) and a speech therapist were involved in the development of the app. They actively participated in the selection of the images for the interface, including backgrounds, color selection, and while creating the audio files containing recordings of the corresponding letters, colors, numbers, and activities. The same child took part in the final testing of the app. ChilDiBu is currently being used by a speech therapist, working with children suffering from Down syndrome, children with Autism and speech disorders. When the experimental exploitation is completed, the results will be summarized, analyzed and published in a scientific article.

\section{MATERIAL AND METHOD}

The widespread availability of smartphones enables professionals to look for new ways to interact with patients and their treatment and support after leaving the hospital. This is possible due to the good software products that can put into practice this enterprise. Nevertheless, the lack of reliable scientific research that can be supported by real results cannot guarantee that one application can be more effective than another. The related work concerning mobile apps' design for children with special educational needs, their evaluations and obtained results will be discussed in this section. It should be pointed out that the presented analysis does not intend to cover all the available scientific research and mobile applications regarding this issue, but only to outline the main features.

The use of mobile applications as a learning tool for children with special educational needs is not a new idea. It has been stated in several studies, e.g. [15], [16], [17]. These studies highlight the benefits of the new technologies used in education and training, since the users (children) have access to information when and where they want, and this information can easily be updated. This is reflected in the wide availability of mobile devices used daily by ordinary people, such as smartphones and tablets.

Apart from being some means of training or entertainment, these devices are also used as therapy aids for various diseases. The mobile devices are presented as an opportunity for supporting mental health for adolescents and as psychotherapy tools support in [18]. For Matthews et al., they can offer the "persistent and continuous access to therapeutic materials and activities outside of the therapy session."

The positive influence when helping children with Autism in their daily lifestyle is unquestionable. A study concerned with the effective impact of the mobile applications $i C a n$ and LINKX Project is conducted in [19].

The advantages of using computer and information technology to educate Autistic children are discussed in [20]. This paper pays attention to the difficulty in finding software products in the children's native language. This example indicates that it is not only in Bulgaria that the access to specialized software products tailored to the abilities and potential of the children with disabilities is limited.

The app Super-Fon for iPad for therapies of phonological disorders in children is presented in [21]. The simple design of that game is based on "the metaphon therapy approach, implementing its phases and levels," and the language to which it is directed is the European Portuguese.

The mobile application Sc@ut for the iPad and iPhone is presented in [22]. It is designed for people with Autism, including children. The simple interface is based on graphical objects (pictograms, images, graphics), and it allows the user to construct sentences to communicate with others. This article also reveals the positive feedback from 13 Autistic children, ranging from 4 to 12 years, and also their parents, concerning the app used.

The mobile application Picaa is presented in [23]. This is a picture-based Apple's iPod and iPhone mobile app for children with Autism. It uses animated and non-animated pictograms. Picaa covers different kinds of activities: puzzle, sorting, association, and exploration. An app for iOS is presented in [24]. It uses two modes of access: Parents and Child. Picaa is a database in which the parent can insert an image and a text to it and the Autistic child can look at them and learn them. The picture-based mobile application BIUTIS for Android [25] is developed for vocabulary learning for children with Autism. Its interface is clear and simple. Some of the images are linked with audio files that contain an audio track for listening.

When discussing an interface for children, attention must be paid to the fact that they perceive the world differently 
from adults. As noted in [26] "little formal research has been done on this topic." This article also mentions another interesting fact, namely, if the children do not approve of (dislike) or fail to understand the interface, then the software one has designed has turned into a failure.

To sum up what has been analyzed so far, it can be concluded that the features a mobile application for children with special educational needs should possess are:

1) Compliance with the ethical standards to protect the child's personal data.

2) Secured environment. The app must provide protection on the Internet if access to the web is used, by requiring authentication (password, username, fingerprint, etc.). The best way to provide software updates or access to relevant information is through web services. In this way, the child will not be at risk of a hacker attack or another unauthorized access.

3) Easy access to all features, modules and/or pages.

4) Friendly and intuitive interface (ease of use).

5) Stability and sustainability, including adequate errors processing while the app is used.

6) Different levels of access to the interface features.

7) All mobile apps have a picture-based interface.

To achieve all these features listed above, different methodologies to develop software suitable for children can be used. Such are: user-centered design, contextual design, participatory design, cooperative design, and others [14]. There are some examples in the literature of good practices for using these different approaches. However, a comparative assessment of the superiority of some of them concerning the design of apps for children with disabilities has not been provided yet.

The conclusion from this research can be that the design, development and evaluation methodology of a mobile application must correspond to the psycho-physical capabilities of the users for which it is intended. If this requirement is not met, the use of this software is likely to be a barrier that may negatively affect the emotional or psychophysical progress of the child. Since in this case these are children with special educational needs, what will be leading in the design of the app ChilDiBu is easy navigation between the separate pages, simplified interface, clear images and objects, and simultaneous stimulation of several senses (e.g., ears and eyes).

The conceptual model of the ChilDiBu app is based on a study of the functional capabilities of existing mobile applications, as well as on a previous author's study on the opinion of children without disabilities concerning such apps. This section presents an approach to designing a mobile application adapted to the abilities and needs of children with special educational needs.

Children are a special group of users. The differences between children and adults as users of software systems are presented in [26]. Brockman et al. divide children into several target groups that are characterized by differences in "dexterity, speech, reading, background knowledge and interaction style." The dexterity of children, regardless of their health or intellectual state, develops with age, and when they fail to handle with some task, they quickly lose interest in it. Speech recognition in the Bulgarian language among children with speech disorders is also a major problem that is currently unresolved and what is more, there are no available literary sources on this issue. Children often cannot read until they are 6 years of age. Therefore, when designing an app interface, it must be based on large and easy to use buttons that are with intuitive design. The children's background knowledge is limited and their experience with a computer device or a software program is based on experiments and errors. Last but not least, based on the study of Resnick et al., the interface discussed here is based on the principle: "Make it as simple as possible - and perhaps even simpler" [27].

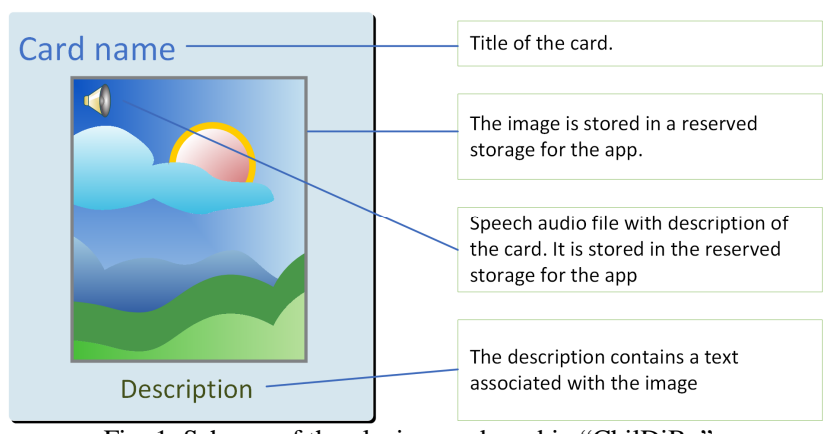

Fig. 1 Schema of the playing card used in "ChilDiBu".

The interface of ChilDiBu is a picture-based app. It is created based on learning multimedia cards (Fig 1). Such playing cardboards are used in real work with children with disorders known as PECS (http://www.pecsusa.com/). Unlike cardboard, the software is more flexible, easy to use and upgrade, and can be installed on a mobile phone, making it easy to carry. The efficacy of using PECS and similar iPad mobile applications for teaching pre-school children with Autism has been demonstrated in [28].

The picture-based approach is widely used in some of the applications discussed in the previous section. The effectiveness of the picture-based systems on the speech development of the children with disorders, in particular children with Autistic spectrum disorders, has been studied and analyzed in [29]. The article by Chen et al. has shown that both types, the speech-generating devices and the picture-based systems, are equally effective and can be applied in clinical practice. This makes me believe that the application development approach proposed in this article is sufficiently effective from a theoretical point of view and it is applicable to the children with disabilities.

The peculiarity of each virtual card (Fig.1) is that it consists of a clear and simple interface. It has a graphic image that relates unequivocally with the visualized object, as well as, a short explanatory text and an audio track that can be played. According to the authors [30] and [31], the children with disabilities have a limitation in communication and speech skills, and they can hardly concentrate on one object. That is why the selection of images was aimed at maximum simplicity and lack of side objects. All this is intended to allow the child to build a clear picture of an object based on three points: image-text-sound. 
For example, if the word "Read" is to be presented through a learning card, it can contain an illustration of an open book and a child, a text that describes it, and the audio file with the pronunciation of the word "read".

Images from the international websites for sharing highquality public domain photos, illustrations, vector graphics Pixabay (http://www.pixabay.com) and Terdpongvector / Freepik (http://www.freepik.com) have been used in most card designs. That images are released under Creative Commons CCO. All of them are processed to fit the overall design concept of the app interface. Some of the images were created in the vector graphics software Inkscape, version 0.92.1 (https://inkscape.org/en/).

The audio files in the ChilDiBu app contain a woman's speech, except for the pronunciation of the letters, made by a nine-year-old child. The records are stereo. They were made with a Praat program, version 6.0.29 (http://www.fon.hum.uva.nl/praat/) in a closed, soundproofed room. The sampling frequency is $44100 \mathrm{~Hz}$, and the used file format is wav. A dynamic microphone with sensitivity: $-80 \mathrm{~dB}+/ 3 \mathrm{~dB}$; frequency response: $100-10,000$ $\mathrm{Hz}$; impedance: $600 \mathrm{ohms}$ was used.

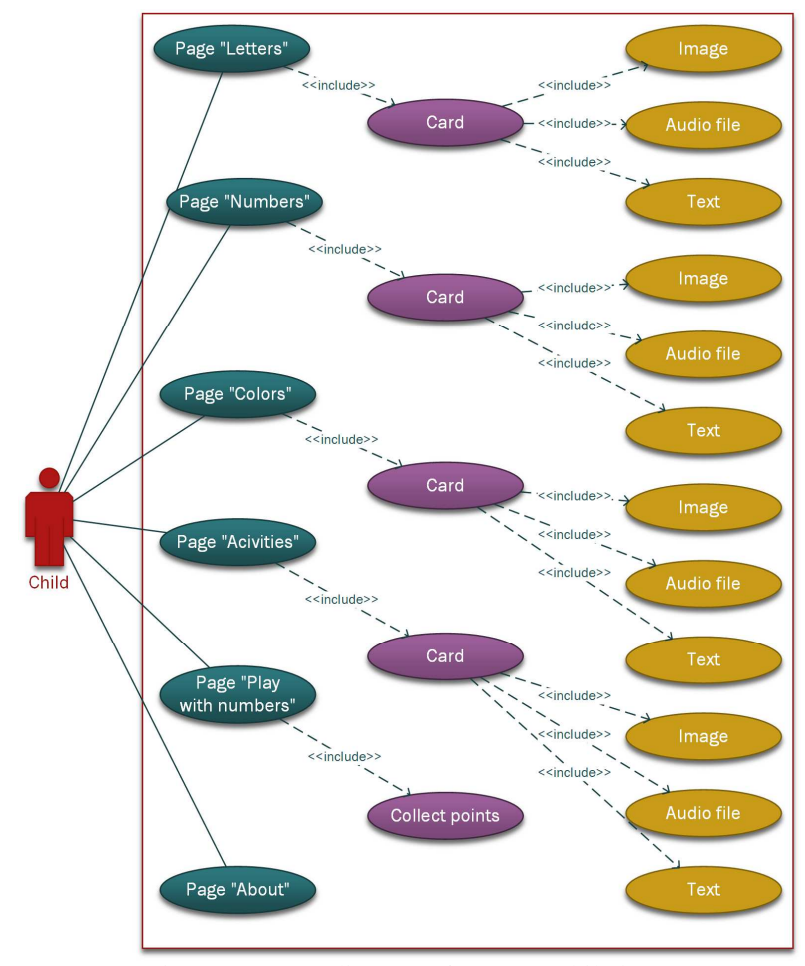

Fig. 2 Use case diagram of an app "ChilDiBu"

The conceptual model of the ChilDiBu application is presented in Fig. 2. The child has access to six pages. Five of them (Letters, Numbers, Colors, Activities, Play with Numbers) are related to his/her development of observation and concentration. One is an informative page (About page). The use case diagram of the app ChilDiBu shows that the cards contain rich multimedia information. The only exception is the page "Play with numbers" where there are tiles with the ten digits from 0 to 9 inclusive. These tiles serve to solve the problem: find and place the correct figure in the right place by collecting points for every correct answer. The aim is to stimulate both the intellectual abilities and the fine motor skills of the fingers of children with special educational needs.

\section{RESULTS AND DISCUSSION}

The mobile application ChilDiBu was developed with an integrated development environment Microsoft Visual Studio Community 2015, Version 14.0.25431 Update 3 and it was tested on the Mobile Emulator 10.0.10586.0 720p 5inch 1GB and Emulator 8.1 WVGA 4inch 512MB. Currently, it can only work on the Windows Mobile 7, 8, 10 mobile operating system. This mobile operating system is selected as a result of a study presented in [32]. It should be noted that this app behaved equally stable and without any difference in the visualization of the interface elements with other Windows Phone emulators that are available to this version of Visual Studio. No errors were found during all tests made by children.

The interface of ChilDiBu is simplified and unified in all the pages. This will allow the children to be confident that once they can work with one of all the app's pages, then they will find it easy to cope with others.

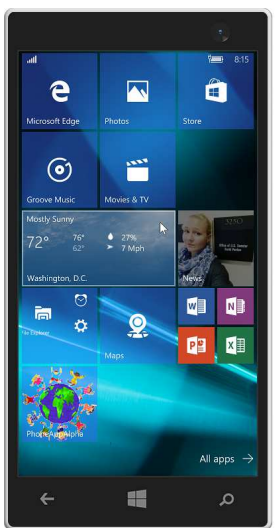

a)

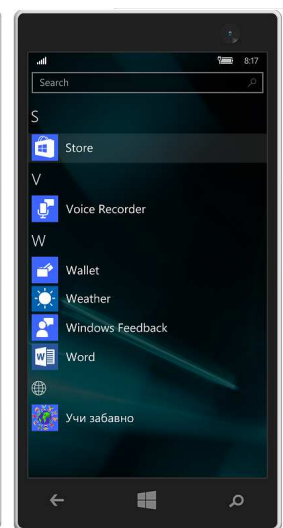

b)

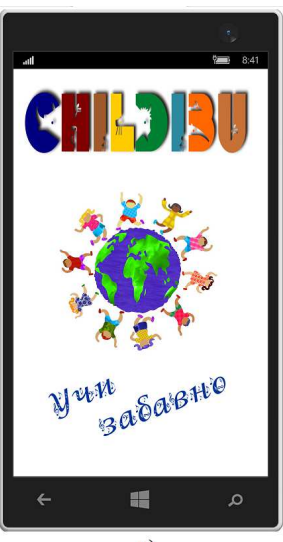

c)
Fig. 3 The screens of "ChilDiBu" (a) the app's tile on the Start page, (b) the app's tile in the list of all applications, and (c) a splash page of the app.

The app ChilDiBu can be started by selecting its tile. It can be found on the start screen of the phone or in the list of all apps (Fig. 3a and Fig. 3b). After launching the app, the splash page is loaded (Fig. 3c). The app allows the use of the interactive cards with the Bulgarian letters ("Letters" page), the numbers up to 20 ("Numbers" page), some of the common colors ("Colors" page), some of the daily activities ("Activities" page) and a puzzle game to arrange of two-digit numbers ("Play with numbers" page). All cards are in a Bulgarian language.

All pages' screens of the app ChilDiBu, together with the main page, are presented in Fig. 4. The user can go back to the main page from all pages using the button Back. The app can be closed with the button Exit. The button About on the main page leads to the page with the author's information and the user help.

The audio files related to the interactive multimedia cards on the "Letters", "Numbers", "Colors" and "Actions" pages are started by double-clicking on their corresponding images. The navigation to each card is possible by touch gestures: flick/swipe. The user can flick and see what the next interactive card is. 


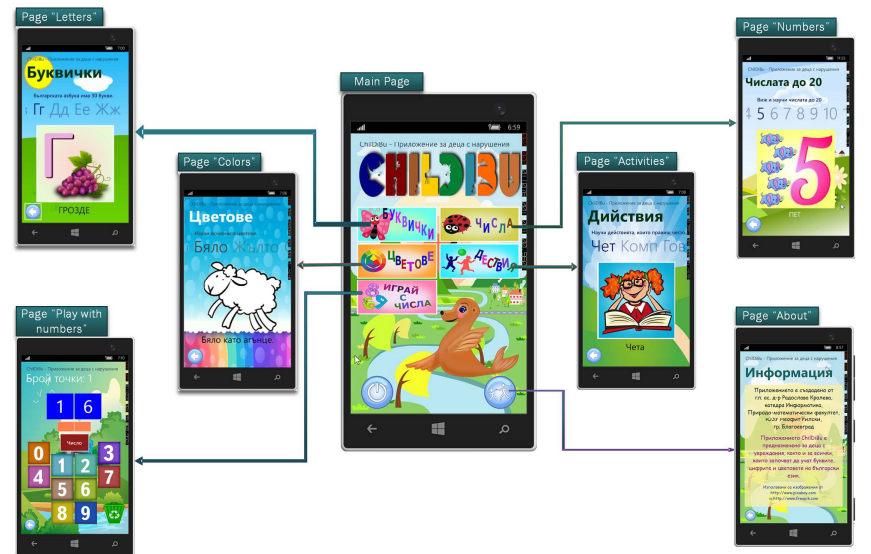

Fig. 4 Architecture overview of the mobile application „ChilDiBu“.

The puzzle game "Play with numbers" is designed to develop the coordination, motoric capabilities, observation skills and resourcefulness in children with special educational needs. The interface design of the game is built in the form of a robot body.

This game does not use stressful sounds when an error is committed. The only way to inform the user about an event is through colors - green (correct) and red (wrong) (Fig. 5). Moving tiles with numbers from 0 to 9 is done by dragging and dropping.

The body, arms and legs of the robot are composed of the digits' tiles from 0 to 9 , and they are painted in different colors.

The robot's eyes are colored in blue (Fig. 5a) and contain the digits of a two-digit number that must be arranged by the child. The mouth of the robot consists of two orange tiles. The tiles of the eyes and the mouth change their color depending on the operation performed by the user. When the child begins to move a digit tile, if the left eye is empty, then the left tile of the robot's mouth turns yellow. If the child can place the correct tile on the robot's left eye, then the eye and the left tile of the mouth are colored green. The same actions are applied to the right eye and the right tile from the robot's mouth. If the number tile is incorrectly placed on one of the robot's eyes, it together with the tile of the mouth below turn red. The robot's neck is composed of the button "Number" (Fig. 5) that can generate a new two-digit number.

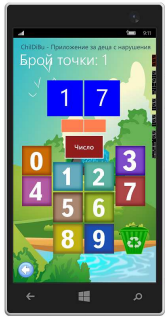

a)

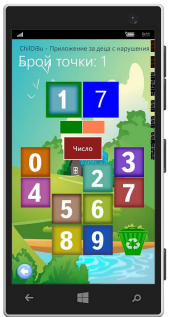

b)

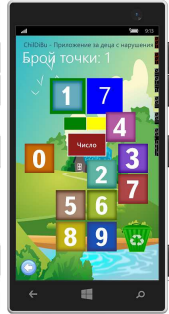

c)

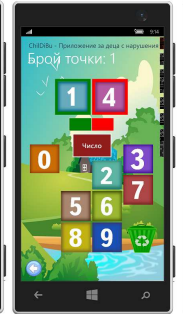

d)

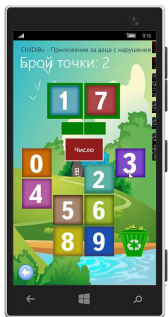

e)
Fig. 5 Views of the page "Play with numbers" and separate screens when it is used.

There is a trash bin in the lower right corner of the page. If the child decides that a tile is unnecessary or he/she wants to return it to the original position, then he/she must take it to the bin and it will return where it was before. When a double-click on the trash bin icon is performed, all the tiles are arranged in their original position.

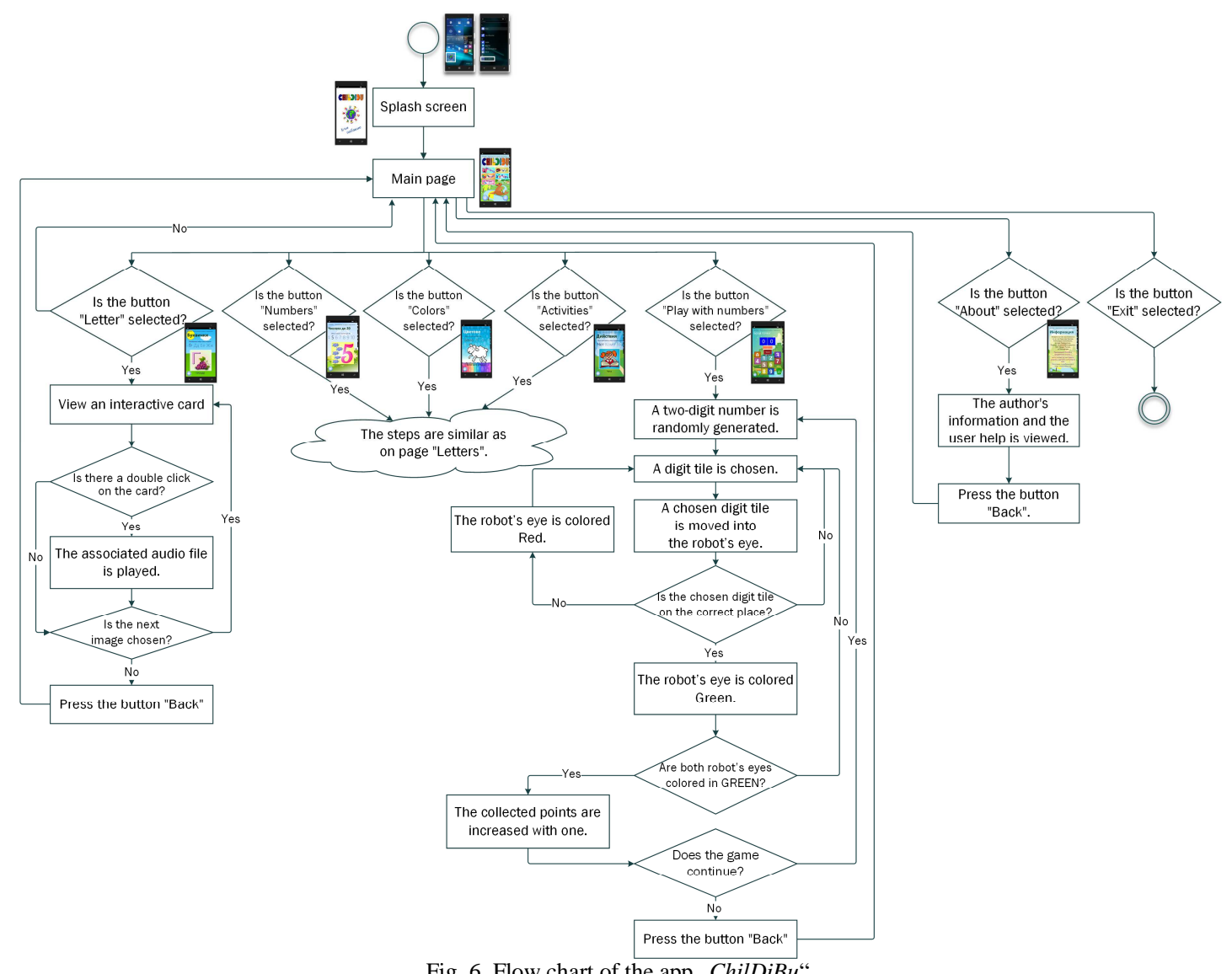

Fig. 6 Flow chart of the app „ChilDiBu“. 
The game "Play with numbers" can count the correctly ordered two-digit numbers. This allows to trace the development of the child's progress when he/she has used the app.

The app's business logic is represented schematically in Fig. 6. Since the steps of page "Letters" are similar to the steps of the pages "Numbers", "Colors" and "Activities", they have been omitted for the sake of brevity. Fig. 6 shows how to use the page "Play with numbers".

\section{CONCLUSION}

A conceptual model and interface of the pilot application for teaching children with communication disorders "ChilDiBu" has been presented in this article. The working language of the interface is Bulgarian as previous studies on this issue have shown that there are no special applications in the Bulgarian language for children with disabilities. This app is based on the rich experience of the author and has benefited from the participation of children. These were the prerequisites for creating applications that fit the needs and desires of the children themselves.

The presented application gives the opportunity to learn the Bulgarian alphabet, the numbers from 0 to 20 , some basic colors and activities from real everyday life using virtual cards for learning. Each card consists of a graphical image, text, and audio file that is started by double-clicking on the card. All audio files are of a woman and a child, and contain a record of pronouncing the individual letter, number, color, or some activity. The text of the card is different for the different groups of cards. The used images are specially selected to meet the requirements of the overall app interface.

The "Play with numbers" page enables children to develop their coordination, observation, and concentration. They also can collect points by arranging digits correctly in the randomly generated numbers. There are no audio files added because some of the disabled children may find some sounds stressful.

The proposed app will be tested with the help of children with disorders in real-life situations. Its effectiveness will be analyzed and the results obtained will be published. The target group is children with different types of disorders such as: Autistic children, with speech disorders, and children without disabilities. The main goal is to establish the effectiveness of the developed application for a larger audience.

The application will be uploaded for free download at the Microsoft Store and will be available for Windows Phone Version 7, 8 and 10 in the near future. At present the author is working on a mobile application version for Android.

\section{ACKNOWLEDGMENT}

I would like to express my sincere gratitude to Dr. Velin Kralev and Assoc. Prof. Dr. Daniela Botseva and Aleksandar Stoimenovski from the SWU "Neofit Rilski", Blagoevgrad, Bulgaria, for their valuable professional advice in the process of development of ChilDiBu, and to Dr. Dafina Kostadinova, from the SWU "Neofit Rilski", Blagoevgrad, Bulgaria, for her constructive criticism regarding the article. I would like to express my appreciation to the reviewers of this article whose expertise and professional recommendation have improved its qualities and significance.

\section{REFERENCES}

[1] G. Kbar and S. Aly, "SMART workplace for persons with Disabilities (SMARTDISABLE)," in Proc. International Conference on Multimedia Computing and Systems (ICMCS), pp. 996-1001, 2014.

[2] S. Edwards, M. Henderson, D. Gronn, A. Scott, M. Mirkhil, "Digital disconnect or digital difference? A socio-ecological perspective on young children's technology use in the home and the early childhood centre," Technology, Pedagogy and Education, vol. 26 (1), pp. 1-17, Jan. 2017.

[3] World Health Organization (2012) Early childhood development and disability: A discussion paper, Donna Phillips, Ed., UNICEF, Switzerland. [Online]. Available: http://apps.who.int/iris/ bitstream/10665/75355/1/9789241504065_eng.pdf

[4] Q. Yu and Z. He, "Comprehensive investigation of temporal and autism-associated cell type composition-dependent and independent gene expression changes in human brains," Scientific Reports, vol. 7 (1), art. num. 4121, Dec. 2017.

[5] C. Grossard, O. Grynspan, S. Serret, A.-L. Jouen, K. Bailly and D. Cohen, "Serious games to teach social interactions and emotions to individuals with autism spectrum disorders (ASD)," Computers and Education, vol. 113, pp. 195-211, Oct. 2017.

[6] S. Baron-Cohen, A. M. Leslie and U. Frith, "Does the autistic child have a theory of mind?," Journal Cognition, vol. 21, pp. 37-46, 1985.

[7] G. R. Hayes, S. Hirano, G. Marcu, M. Monibi, D. H. Nguyen and M. Yeganyan, "Interactive visual supports for children with Autism," Personal and Ubiquitous Computing, vol. 14, pp. 663-680, 2010.

[8] S. Judge, K. Puckett and B. Cabuk, "Digital equity: New findings from the early childhood longitudinal study," Journal of Research on Technology in Education, vol. 36 (4), pp. 383-96, 2004.

[9] S. Jackson (2011) "Learning, digital media, and creative play in early childhood," Spotlight on Digital Media and Learning (blog), March 24. Chicago, IL: MacArthur Foundation. [Online]. Available: http://spotlight.macfound.org/featured-stories/entry/ learning-digitalmedia-and-creative-play-in-early-childhood

[10] D. McNaughton and J. Light, "The iPad and mobile technology revolution: Benefits and challenges for individuals who require augmentative and alternative communication," Journal Augmentative and Alternative Communication, vol. 29 (2), pp. 107-111, 2013.

[11] R. Cowen, M. Mitchel, A. E. Hare-Harris and B. R. King, "Application of n-gram prediction and Brown's Stages of Syntactic and Morphological Development to design augmentative and alternative communication for children with autism," in Proc. at 5th Annual Susquehanna Valley Undergraduate Research Symposium, SVURS 2015, Bucknell University, Lewisburg, PA. (Poster), August 2015 .

[12] S. Baldassarri, J. M. Rubio, M. G. Azpiroz and E. Cerezo, "AraBoard: A multiplatform alternative and augmentative communication tool," Journal Procedia Computer Science, vol. 27, pp. 197-206, 2014.

[13] R. Dunn and A. Vyshedskiy, "Mental imagery therapy for Autism (MITA) - An early intervention computerized brain training program for children with ASD," Autism Open Access, vol. 5 (3), p. 153, 2015.

[14] R. Kraleva, "Designing an Interface for a mobile application based on children's opinion," International Journal of Interactive Mobile Technologies (iJIM), vol. 11 (1), pp. 53-70, March 2017.

[15] G. Kbar, A. Bhatia and M. H. Abidi, "Smart unified interface for people with disabilities at the work place," in Proc. International Conference on Innovations in Information Technology (IIT), 2015.

[16] V. Sondhi and A. Devgan, "Translating technology into patient care: Smartphone applications in paediatric health care," Medical Journal Armed Forces India, vol. 69 (2), pp. 156-161, 2013.

[17] J. Vlachou and A. Drigas, "Mobile technology for students \& adults with Autistic Spectrum Disorders (ASD)," International Journal of Interactive Mobile Technologies (iJIM), vol. 11 (1), pp. 4-17, 2017.

[18] M. Matthews, G. Doherty, D. Coyle and J. Sharry, "Designing mobile applications to support mental health interventions," J Lumsden, Ed., Handbook of Research on User Interface Design and Evaluation for Mobile Technology, IGI Global, pp. 635-656, 2008.

[19] S. Joshi, A. Biyani, R. Kamdar, A. Bhajan and A. Singh, "Review on mobile application for children suffering from Autism," International Journal of Advanced Research in Computer Science and Software Engineering, vol. 5 (5), pp. 831-834, May 2015. 
[20] J. Khattak, M. Munawar, U. Sajjad and S. Shahid, "Designing digital aids for children with Autism in Pakistan: Challenges and possibilities," International Workshop of Computing in Development (CHI 2016), San Jose, USA. [Online]. Available: http://igw.tuwien.ac.at/chi16-autismtechnology/attachments/ MunawarEtAl.pdf

[21] R. N. Madeira, P. Macedo, S. Reis and J. Ferreira, "Super-Fon mobile entertainment to combat phonological disorders in children," in Proc. of ACE '14 Proceedings of the 11th Conference on Advances in Computer Entertainment Technology, article No. 53, Funchal, Portugal — November 11 - 14, 2014.

[22] M. J. Rodríguez-Fórtiz, A. Fernández-López and M. L. Rodríguez, "Mobile communication and learning applications for Autistic people, Autism Spectrum Disorders - from genes to environment," T. Williams, Ed., Autism Spectrum Disorders - From Genes to Environment, InTechOpen, Sep 2011.

[23] Á. Fernández-López, M. J. Rodríguez-Fórtiz, M. L. RodríguezAlmendros and M. J. Martínez-Segura, "Mobile learning technology based on iOS devices to support students with special education needs," Computers \& Education, vol. 61, pp. 77-90, 2013.

[24] M. Z. Z. Aziz, S. A. C. Abdullah, S. F. S. Adnan and L. Mazalan, "Educational app for children with Autism Spectrum Disorders (ASDs)," in Proc. International Conference on Robot PRIDE 20132014 - Medical and Rehabilitation Robotics and Instrumentation, ConfPRIDE 2013-2014, Procedia Computer Science, vol. 42, pp. 70 $-77,2014$.

[25] E. Husni, "Mobile applications BIUTIS: Let's study vocabulary learning as a media for children with Autism," Procedia Technology, vol. 11, pp. $1147-1155,2013$.
[26] A. Bruckman and A. Bandlow, "HCI for kids," J. Jacko and A. Sears, Ed., The Human-Computer Interaction Handbook: Fundamentals, Evolving Technologies, and Emerging Applications, Lawrence Erlbaum and Associates, pp. 428-440, 2003.

[27] M. Resnick and B. Silverman, "Some reflections on designing construction kits for kids," in Proc. Conference on Interaction Design and Children, Boulder, CO, pp. 117-122, 2005.

[28] M. M. Agius and M. Vance, "A comparison of PECS and iPad to teach requesting to preschoolers with Autistic Spectrum Disorders," Augmentative and Alternative Communication, vol. 32 (1), 2016.

[29] C.-H. Chen, C.-P. Wang, I-J. Lee, C. C.-C. Su, "Speech-generating devices: effectiveness of interface design - a comparative study of autism spectrum disorders," Springerplus, vol. 5 (1): 1682, 2016.

[30] E. R. Lorah, M. Tincani, J. Dodge, S. Gilroy, A. Hickey, and D. Hantula, "Evaluating picture exchange and the iPad® as a speech generating device to teach communication to young children with autism," Journal of Developmental and Physical Disabilities, vol. 25, pp. 637-649, 2013

[31] J. Sigafoos, M. F. O'Reilly, G. E. Lancioni and D. Sutherland "Augmentative and alternative communication for individuals with autism spectrum disorder and intellectual disability," Journal Current Developmental Disorders Reports, vol. 1 (2), pp. 51-57, June 2014

[32] R. Kraleva, A. Stoimenovski, D. Kostadinova, V. Kralev, "Investigating the opportunities of using mobile learning by young children in Bulgaria," International Journal of Computer Science and Information Security, vol. 14 (4), pp. 51-55, 2016. 